Supporting Information for:

\title{
Mechanistic Evidence for a Ring-Opening Pathway in the Pd-catalyzed Direct Arylation of Benzoxazoles
}

\author{
Rafael S. Sánchez and Fedor A. Zhuravlev*
}

Technical University of Denmark, Building 201, Kemitorvet, DK-2800 Kgs. Lyngby, Denmark

\section{Experimental details}

General Methods. All reactions were carried out under argon atmosphere. 5-substituted benzoxazoles were either purchased from Aldrich or synthesized as described elsewhere ${ }^{1}$. All solvents were either distilled or vacuum-transferred prior to use. Acetone and DMF were dried over activated $4 \AA$ molecular sieves. ${ }^{1} \mathrm{H}(300 \mathrm{MHz})$ and ${ }^{13} \mathrm{C}(75$ $\mathrm{MHz}$ ) NMR spectra were recorded using deuterated chloroform unless otherwise noted, and signal positions were referenced relative to the signals for $\mathrm{CHCl}_{3}$ (7.25) and $\mathrm{CDCl}_{3}$ (77.0), respectively. All compounds exhibited clean ${ }^{1} \mathrm{H} N M R$ and ${ }^{13} \mathrm{C}$ spectra and showed one spot on TLC analysis. TLC analyses were performed on Merck aluminumbacked F254 silica gel plates, using UV light. Column chromatography was performed using Amicon Matrex silica gel $(35-70 \mu \mathrm{m})$. Quantitative GC analysis was performed on a Shimadzu GC-2010 gas chromatograph using Supelco Equity-1 Capillary column. 2,3dimethylnaphthalene was used as internal reference.

Kinetic Studies. A dry $25 \mathrm{~mL}$ flask was charged with $\mathrm{Cs}_{2} \mathrm{CO}_{3}(2.0 \mathrm{mmol})$ and 5substituted benzoxazole $(1.0 \mathrm{mmol})$. To this, $1 \mathrm{~mL}$ of a stock solution of $\mathrm{Pd}(\mathrm{OAc})_{2}(0.025$ $\mathrm{mmol}) / \mathrm{PPh}_{3}(0.1 \mathrm{mmol})$, and 2,3-dimethylnaphthalene $(1.0 \mathrm{mmol})$ was added, followed by the addition of $\mathrm{Phl}(20 \mathrm{mmol})$ and DMF to a total volume of $10 \mathrm{~mL}$. The flask was thermostated at $100{ }^{\circ} \mathrm{C}$ and the reaction progress was monitored by taking aliquots and subjecting them to the $\mathrm{GC}$ analysis.

\section{Kinetic Data}

Table S1. Arylation of Benzoxazole

\begin{tabular}{|l|l|l|}
\hline Time (sec) & $\ln \frac{[\text { benzoxazole }]_{0}}{[\text { benzoxazole }]_{t}}$ & Conversion (\%) \\
\hline 0 & 0 & 0 \\
1800 & 0.12557 & 11.8 \\
3600 & 0.21134 & 19.0 \\
5400 & 0.26529 & 23.3 \\
9000 & 0.4065 & 33.4 \\
12600 & 0.54224 & 41.9 \\
16200 & 0.661 & 48.4 \\
19800 & 0.78933 & 54.6 \\
\hline
\end{tabular}




\begin{tabular}{|l|l|l|}
\hline 23400 & 0.90478 & 59.5 \\
27000 & 1.03617 & 64.5 \\
30600 & 1.16032 & 68.7 \\
\hline
\end{tabular}

$k_{H}^{a p p}=3.93 \times 10^{-5} \pm 8 \times 10^{-7} ; R^{2}=0.996$

Table S2. Arylation of 5-methylbenzoxazole

\begin{tabular}{|l|l|l|}
\hline Time (sec) & $\ln \frac{[5-\text { methylbenzoxazole }]_{0}}{[5-\text { methylbenzoxazole }]_{t}}$ & \\
\hline 0 & 0 & 0 \\
1800 & 0.08258 & 7.92646 \\
3600 & 0.1421 & 13.24657 \\
5400 & 0.20105 & 18.21319 \\
9000 & 0.26359 & 23.17104 \\
12600 & 0.31922 & 27.32874 \\
16200 & 0.44448 & 35.88419 \\
19800 & 0.50948 & 39.91917 \\
23400 & 0.56169 & 42.9753 \\
27000 & 0.66189 & 48.41248 \\
\hline
\end{tabular}

$k_{M e}^{a p p}=2.31 \times 10^{-5} \pm 9 \times 10^{-7} ; R^{2}=0.989$

Table S3. Arylation of 5-chlorobenzoxazole

\begin{tabular}{|l|l|l|}
\hline Time $(\mathrm{sec})$ & $\ln \frac{[5-\text { chlorobenzoxazole }]_{0}}{[5-\text { chlorobenzoxazole }]_{t}}$ & \\
\hline 0 & 0 & 0 \\
240 & 0.02022 & 2.00151 \\
540 & 0.05398 & 5.2552 \\
1140 & 0.06617 & 6.40257 \\
2040 & 0.11913 & 11.23062 \\
2940 & 0.16443 & 15.16214 \\
4740 & 0.26149 & 23.00979 \\
\hline
\end{tabular}

$k_{C l}^{\text {app }}=5.33 \times 10^{-5} \pm 2.0 \times 10^{-6} ; R^{2}=0.992$

Table S4. Arylation of 5-methoxybenzoxazole

\begin{tabular}{|l|l|l|}
\hline Time (sec) & $\ln \frac{[5-\text { methoxybenzoxazole }]_{0}}{[5-\text { methoxybenzoxazole }]_{t}}$ & \\
\hline 1800 & 0.07082 & 6.8367 \\
3600 & 0.11157 & 10.55696 \\
5400 & 0.14766 & 13.72795 \\
9000 & 0.20792 & 18.77275 \\
12600 & 0.26405 & 23.20623 \\
16200 & 0.32259 & 27.57272 \\
19800 & 0.36887 & 30.84817 \\
23400 & 0.43884 & 35.52129 \\
27000 & 0.47261 & 37.66279 \\
30600 & 0.53558 & 41.46676 \\
\hline
\end{tabular}


$k_{\text {OMe }}^{\text {app }}=1.58 \times 10^{-5} \pm 3 \times 10^{-7} ; R^{2}=0.997$

Table S5. Arylation of 5-nitrobenzoxazole

\begin{tabular}{|l|l|l|}
\hline Time (sec) & $\ln \frac{[5-\text { nitrobenzoxazole }]_{0}}{[5-\text { nitrobenzoxazole }]_{t}}$ & \\
\hline 0 & 0 & 0 \\
300 & 0.51622 & 40.3229 \\
600 & 0.85066 & 57.28655 \\
900 & 1.216 & 70.35856 \\
1200 & 1.50468 & 77.79107 \\
1500 & 1.80088 & 83.48473 \\
1800 & 2.10524 & 87.81838 \\
2100 & 2.32996 & 90.27008 \\
2400 & 2.86291 & 94.28979 \\
2700 & 3.17908 & 95.8376 \\
3000 & 3.86923 & 97.91256 \\
\hline
\end{tabular}

$k_{\mathrm{NO}_{2}}^{a p p}=0.00118 \pm 4.21 \times 10^{-5} ; R^{2}=0.989$

\section{Determination of KIE at C(2) using ${ }^{1} \mathrm{H}$-Detected Isotopic Fractionation}

As an example procedure a mixture of 5-methylbenzoxazole (2.31, $17.37 \mathrm{mmol}), \mathrm{Phl}$ $(14.0 \mathrm{~g}, 69.4 \mathrm{mmol})$, cesium carbonate $(11.30 \mathrm{~g}, 34.70 \mathrm{mmol}), \mathrm{Pd}(\mathrm{OAc})_{2}(0.112 \mathrm{~g}, 0.5$ mmol), $\mathrm{PPh}_{3}(0.525 \mathrm{~g}, 2.0 \mathrm{mmol})$, and 2,3-dimethylnaphthaline (GC standard) in dry DMF was heated to $100 \pm 1{ }^{\circ} \mathrm{C}$ under Ar with vigorous stirring. Aliquots were periodically removed, and the progress of the reaction was analyzed by GC. When the conversion reached $80 \%$ the reaction was stopped and 5-methylbenzoxazole was re-isolated and purified by sublimation. ${ }^{1} \mathrm{H}$ NMR spectrum was integrated $\left(\mathrm{C}(2)-\mathrm{H}\right.$ and $\mathrm{CH}_{3}$ signals) and the isotopomeric ratio was estimated as described in reference 20 giving $\mathrm{KIE}=1.07 \pm 0.09$ $(F=0.80, \mathrm{R} / \mathrm{Ro}=1.12)$.

\section{5-methylbenzoxazole, initial}
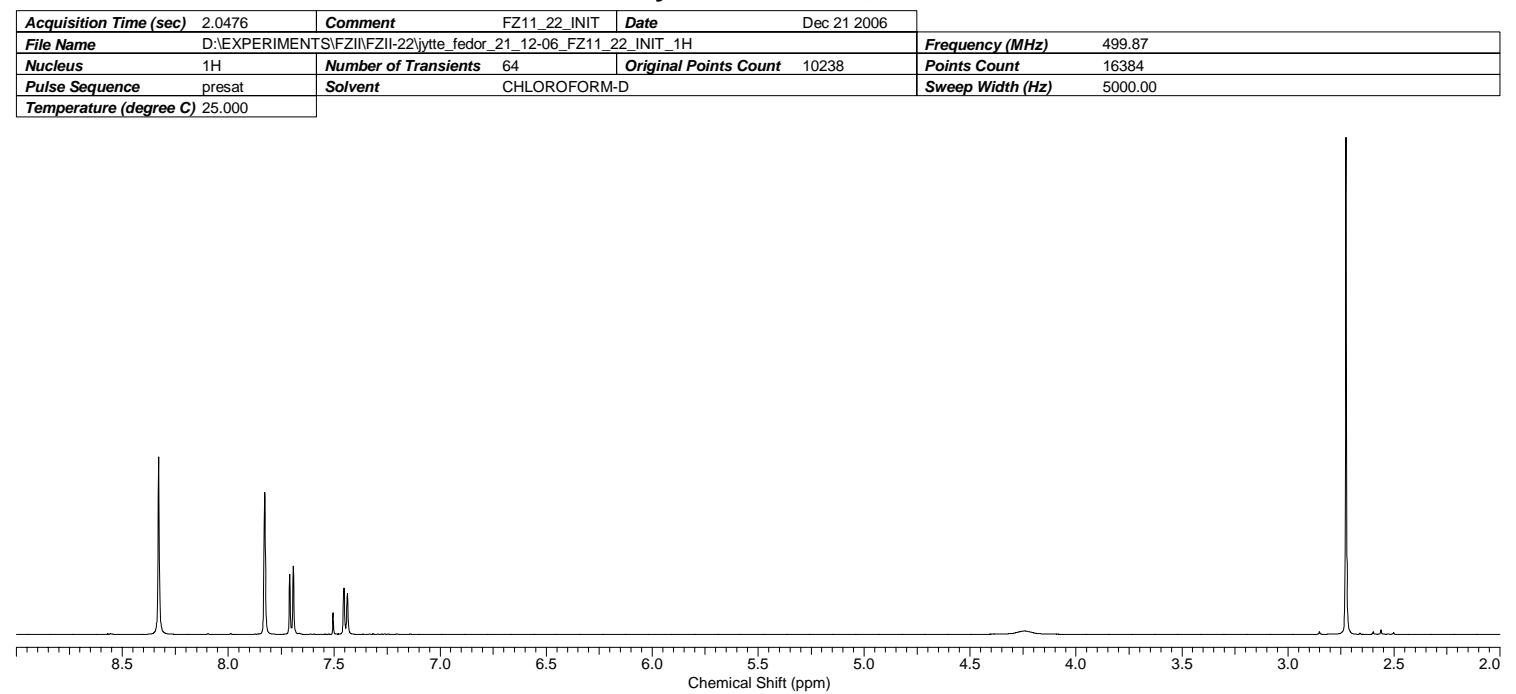


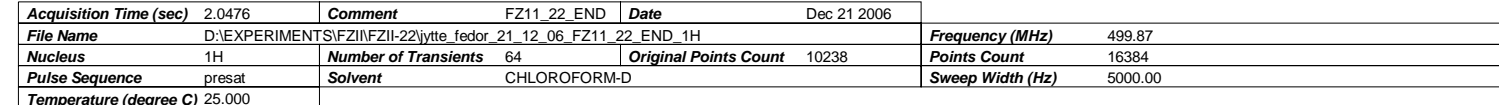

Temperature (degree C) 25.000

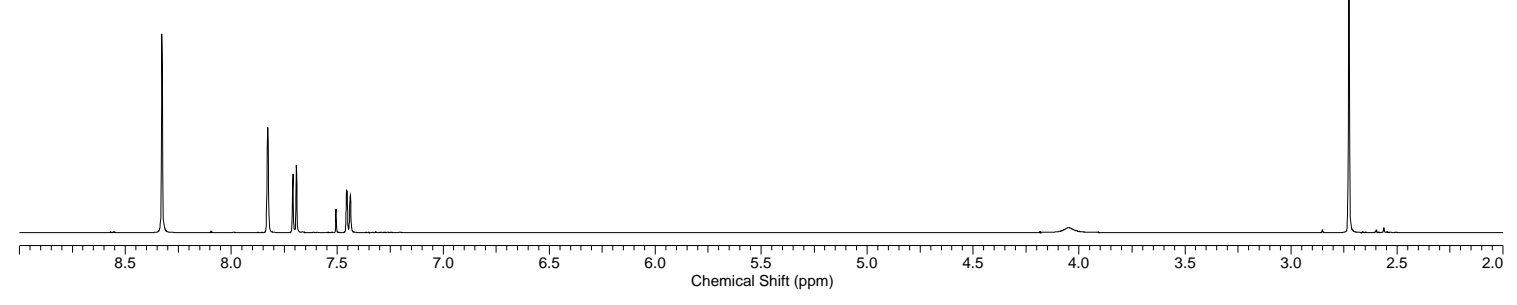

The analytical derivation of the rate law for the proposed mechanism

The analytical rate law was derived using the linear network method (Reference S2). Assuming the quasi-equilibrium conditions for the phosphine dissociation and the proton transfer steps, and kinetically lumping intermediates 2 and 3 the catalytic cycle can be represented as follows:

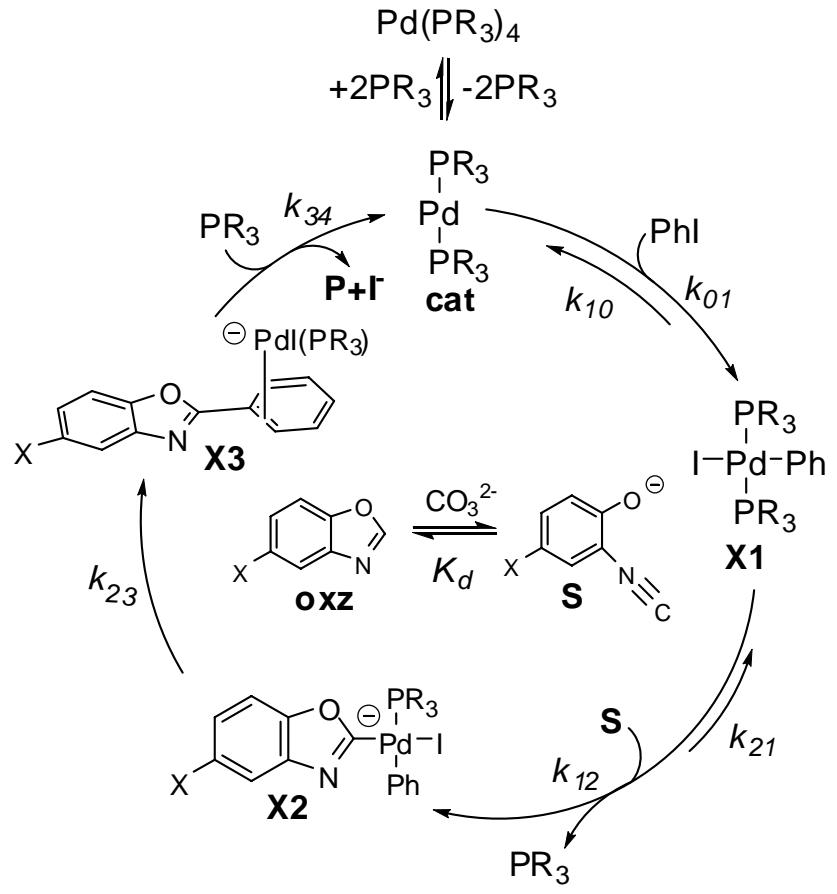


The quasi-equilibrium conditions yield:

$C_{0}=\left[P d\left(P R_{3}\right)_{2}\right]=K_{P} \frac{\left[P d\left(P R_{3}\right)_{4}\right]}{\left[P R_{3}\right]^{2}}=K_{P} \frac{C_{P d}}{C_{P R_{3}}^{2}} ; \quad K_{d}^{\prime}=\frac{[S]\left[\mathrm{HCO}_{3}^{-}\right]}{[\mathrm{oxz}]\left[\mathrm{CO}_{3}^{2-}\right]} ; \quad C_{s}=K_{d} C_{o x z}$

Following the linear pathway formalism, the rate can be written as:

$r_{p}=\Lambda_{04} C_{0}-\Lambda_{40} C_{p}$

Using indices 0 and 4 in $\lambda$ coefficients for the catalyst (cat) and the product (P), and ${ }_{1,2,3}$ for the trace-level intermediates $\mathrm{X} 1, \mathrm{X} 2$, and $\mathrm{X} 3$ respectively, the segment coefficient matrix can be written as:

$$
\begin{aligned}
& \left(\begin{array}{cccc}
1 & \lambda_{12} & \lambda_{23} & \lambda_{34} \\
\lambda_{10} & 1 & \lambda_{23} & \lambda_{34} \\
\lambda_{10} & \lambda_{21} & 1 & \lambda_{34} \\
\lambda_{10} & \lambda_{21} & \lambda_{32} & 1
\end{array}\right) \\
& \Lambda_{04}=\frac{\lambda_{01} \lambda_{12} \lambda_{23} \lambda_{34}}{D_{04}} \quad \Lambda_{40}=\frac{\lambda_{10} \lambda_{21} \lambda_{32} \lambda_{43}}{D_{04}} ; \\
& D_{04}=\lambda_{12} \lambda_{23} \lambda_{34}+\lambda_{10} \lambda_{23} \lambda_{34}+\lambda_{10} \lambda_{21} \lambda_{34}+\lambda_{10} \lambda_{21} \lambda_{32} \\
& \lambda_{01}=k_{01} C_{P h I} \quad \lambda_{12}=k_{12} C_{S} \quad \lambda_{23}=k_{23} \quad \lambda_{34}=k_{34} C_{P R_{3}} \\
& \lambda_{10}=k_{10} \quad \lambda_{21}=k_{21} C_{P R_{3}} \quad \lambda_{32}=0 \quad \lambda_{43}=0 \\
& r_{p}=\frac{\lambda_{01} \lambda_{12} \lambda_{23}}{\lambda_{12} \lambda_{23}+\lambda_{10} \lambda_{23}+\lambda_{10} \lambda_{21} C_{0}=\frac{k_{01} k_{12} k_{23} C_{P h I} C_{S}}{k_{12} k_{23} C_{S}+k_{10} k_{23} C_{P h I}+k_{10} k_{21} C_{P R_{3}} C_{P h I}} C_{0}} \\
& r_{p}=\frac{k_{01} k_{12} k_{23} C_{P h I} C_{S} C_{0}}{k_{12} k_{23} C_{S}+k_{10} k_{23} C_{P h I}+k_{10} k_{21} C_{P R_{3}} C_{P h I}} \\
& =\frac{k_{01} k_{12} C_{P h I} C_{S} k_{23} C_{0}}{\left(k_{10} k_{21}, k_{10} k_{23}, k_{12} k_{23}\right) \cdot\left(C_{P h I} C_{P R_{3}}, C_{P h I} C_{S}\right)} \\
& =\frac{k_{01} k_{12} C_{P h I} C_{S}}{\left\|\left(k_{10} k_{21}, k_{10} k_{23}, k_{12} k_{23}\right)\right\| \cdot\left\|\left(C_{P h I} C_{P R_{3}}, C_{P h I} C_{S}\right)\right\| \cdot \cos \theta} \cdot k_{23} C_{0} \\
& =\frac{k_{01} k_{12}}{\sqrt{\left(k_{10} k_{21}\right)^{2}+\left(k_{10} k_{23}\right)^{2}+\left(k_{12} k_{23}\right)^{2}}} \cdot \frac{C_{P h I} C_{S}}{\sqrt{\left(C_{P h I} C_{P R_{3}}\right)^{2}+C_{P h I}^{2}+C_{S}^{2}}} \cdot \sec \theta \cdot k_{23} \cdot C_{0} \\
& =\frac{k_{01} k_{12} k_{23} K_{P}}{\sqrt{\left(k_{10} k_{21}\right)^{2}+\left(k_{10} k_{23}\right)^{2}+\left(k_{12} k_{23}\right)^{2}}} \cdot K_{d} \cdot \frac{C_{P h I} C_{0 x z} C_{P d}}{C_{P R_{3}}^{2} \sqrt{\left(C_{P h I} C_{P R_{3}}\right)^{2}+C_{P h I}^{2}+C_{S}^{2}}} \cdot \sec \theta
\end{aligned}
$$

where $\theta$ is the angle between $\left(k_{10} k_{21}, k_{10} k_{23}, k_{12} k_{23}\right)$ and $\left(C_{P h I} C_{P R_{3}}, C_{P h I} C_{S}\right)$ in the Euclidean space.

The rate constant can be written in the compact form as:

$k^{o b s}=k_{A r} K_{d}$ 


\section{Computational Details}

All calculations were performed using Turbomole 5.7 suite of programs. Geometry optimizations and transition state search were performed at BP86/TZVP level in gas phase. All calculations used resolution of identity approximation, accurate cutoffs (keywords \$scfconv 8, \$denconv 1d-7 and -gcart 4) and fine integration grid (m4). Vibrational analysis performed on the converged structures showed only positive normal modes for intermediates 1, $\mathbf{P h P d}\left(\mathrm{PH}_{3}\right)_{2} \mathrm{I}, \mathbf{2}, 3, \mathrm{PH}_{3}$ and 2-phenylbenzoxazole:PdI( $\left.\mathrm{PH}_{3}\right)^{-}$ and a single imaginary frequency for the corresponding transition states. The Xmol .xyz coordinates, total energies (Hartree) and imaginary vibrational frequencies for selected structures are listed below.

\begin{tabular}{|l|rrrr|}
\hline 1 & $\mathrm{C}$ & 0.3645499 & -0.4764365 & 0.0000000 \\
$\mathrm{C}$ & -1.0152458 & 0.0369578 & 0.0000000 \\
$\mathrm{C}$ & -1.0692871 & 1.4900190 & -0.0000000 \\
$\mathrm{C}$ & 0.0558277 & 2.2994267 & -0.0000000 \\
$\mathrm{C}$ & 1.3573986 & 1.7527061 & 0.0000000 \\
$\mathrm{C}$ & 1.4892667 & 0.3635132 & 0.0000000 \\
& $\mathrm{H}$ & -2.0719872 & 1.9266664 & 0.0000000 \\
& $\mathrm{H}$ & -0.0681886 & 3.3885164 & -0.0000000 \\
$\mathrm{H}$ & 2.2399872 & 2.3949951 & 0.0000000 \\
& $\mathrm{H}$ & 2.4795699 & -0.1010396 & -0.0000000 \\
& $\mathrm{O}$ & -2.0390867 & -0.6988950 & 0.0000000 \\
& $\mathrm{C}$ & 0.6686778 & -3.0229491 & -0.0000000 \\
& $\mathrm{~N}$ & 0.5561146 & -1.8444010 & -0.0000000 \\
\hline & & & & \\
\hline
\end{tabular}

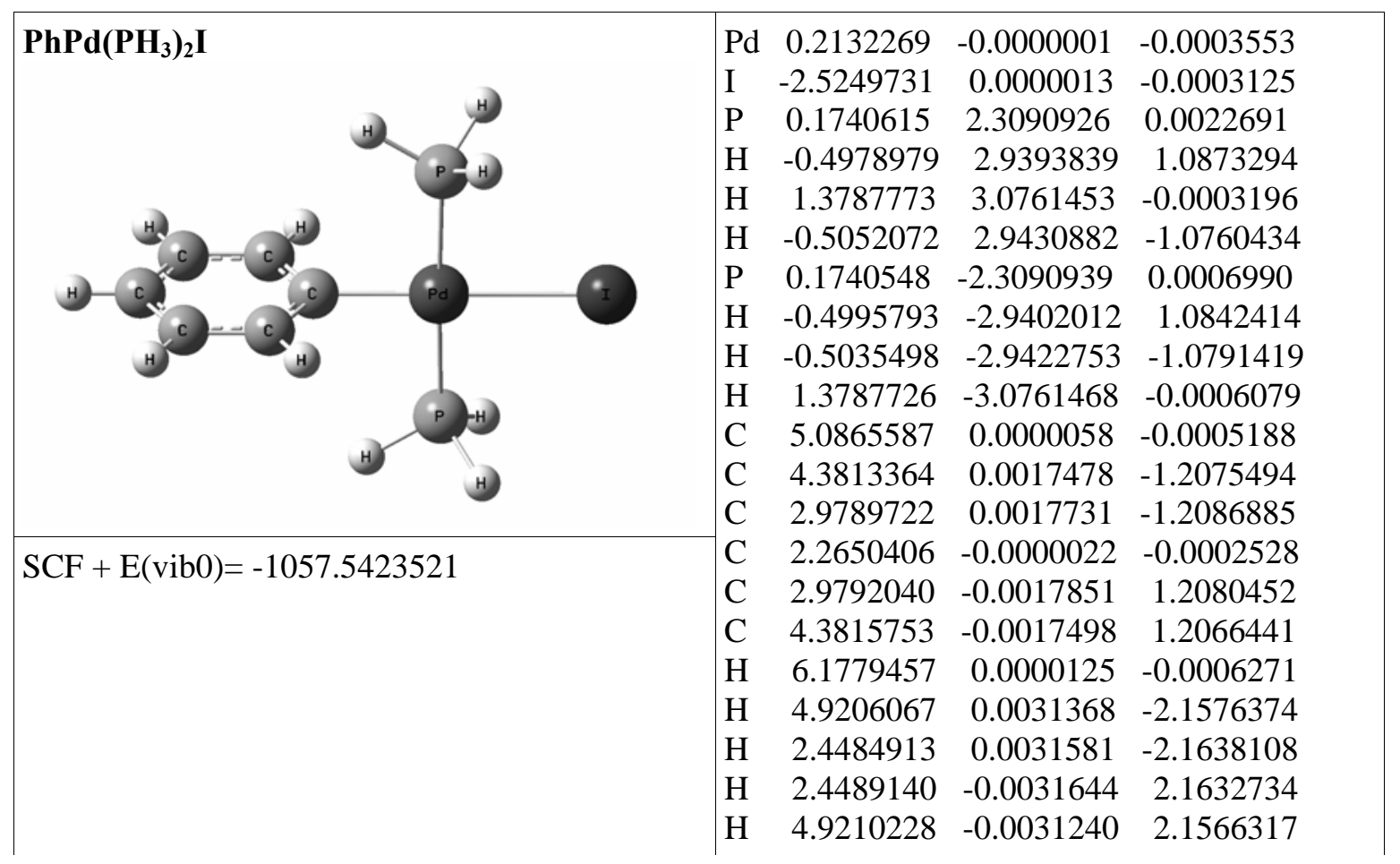




\begin{tabular}{|l|lrrr|}
\hline $\mathbf{P H}_{3}$ & $\mathrm{P}$ & 0.0703439 & 0.0000019 & 0.0000025 \\
\hline & $\mathrm{H}$ & -0.7204985 & 0.1186360 & -1.1942971 \\
& $\mathrm{H}$ & -0.7205205 & -1.0936493 & 0.4943545 \\
& $\mathrm{H}$ & -0.7205705 & 0.9749550 & 0.6998652 \\
& & & & \\
\hline
\end{tabular}

\begin{tabular}{|c|c|c|c|}
\hline 2 & 0.9953332 & 4.4833403 & 0.0011516 \\
\hline (1) है (c) & $\begin{array}{lr}\mathrm{C} & 1.0556086 \\
\mathrm{C} & -0.0722343 \\
\mathrm{C} & -1.3739713 \\
\mathrm{C} & -1.5130255 \\
\mathrm{C} & -0.3807870 \\
\mathrm{H} & 2.0568376 \\
\mathrm{H} & 0.0495089 \\
\mathrm{H} & -2.2536648 \\
\mathrm{H} & -2.5010854 \\
\mathrm{O} & 2.0244405 \\
\mathrm{C} & -0.3307127 \\
\mathrm{~N} & -0.5278411 \\
\mathrm{Pd} & 0.2127464 \\
\mathrm{I} & 1.0288293 \\
\mathrm{P} & 2.4820083 \\
\mathrm{H} & 3.3357626 \\
\mathrm{H} & 2.6529316 \\
\mathrm{H} & 3.3374827 \\
\mathrm{C} & -4.4593395 \\
\mathrm{C} & -3.7898632 \\
\mathrm{C} & -2.4507372 \\
\mathrm{C} & -1.7561693 \\
\mathrm{C} & -2.4368721 \\
\mathrm{C} & -3.7761804 \\
\mathrm{H} & -5.5035807 \\
\mathrm{H} & -4.3102582 \\
\mathrm{H} & -1.9462446 \\
\mathrm{H} & -1.9209112 \\
\mathrm{H} & -4.2856935\end{array}$ & $\begin{array}{c}5.9282073 \\
6.7323912 \\
6.1843441 \\
4.8004024 \\
3.9681680 \\
6.3655048 \\
7.8203231 \\
6.8292600 \\
4.3343148 \\
3.7422789 \\
1.4405117 \\
2.6039212 \\
-0.4260698 \\
-3.0002898 \\
0.3402304 \\
-0.0475464 \\
1.7665426 \\
-0.0486816 \\
-1.8425115 \\
-1.6124254 \\
-1.1986279 \\
-1.0172405 \\
-1.2422206 \\
-1.6552363 \\
-2.1639111 \\
-1.7544375 \\
-1.0263431 \\
-1.1058865 \\
-1.8314904\end{array}$ & $\begin{array}{c}-0.0003314 \\
-0.0047104 \\
-0.0080933 \\
-0.0068782 \\
-0.0024148 \\
0.0022166 \\
-0.0056192 \\
-0.0115548 \\
-0.0094014 \\
0.0050814 \\
0.0005077 \\
-0.0013106 \\
0.0011370 \\
0.0004131 \\
0.0001395 \\
-1.0761435 \\
0.0009470 \\
1.0746642 \\
0.0002195 \\
1.2064023 \\
1.2059310 \\
0.0007949 \\
-1.2049218 \\
-1.2057596 \\
0.0000018 \\
2.1576750 \\
2.1588568 \\
-2.1574968 \\
-2.1571910\end{array}$ \\
\hline $\mathrm{SCF}+\mathrm{E}(\mathrm{vib} 0)=-1113.6254633$ & & & \\
\hline
\end{tabular}




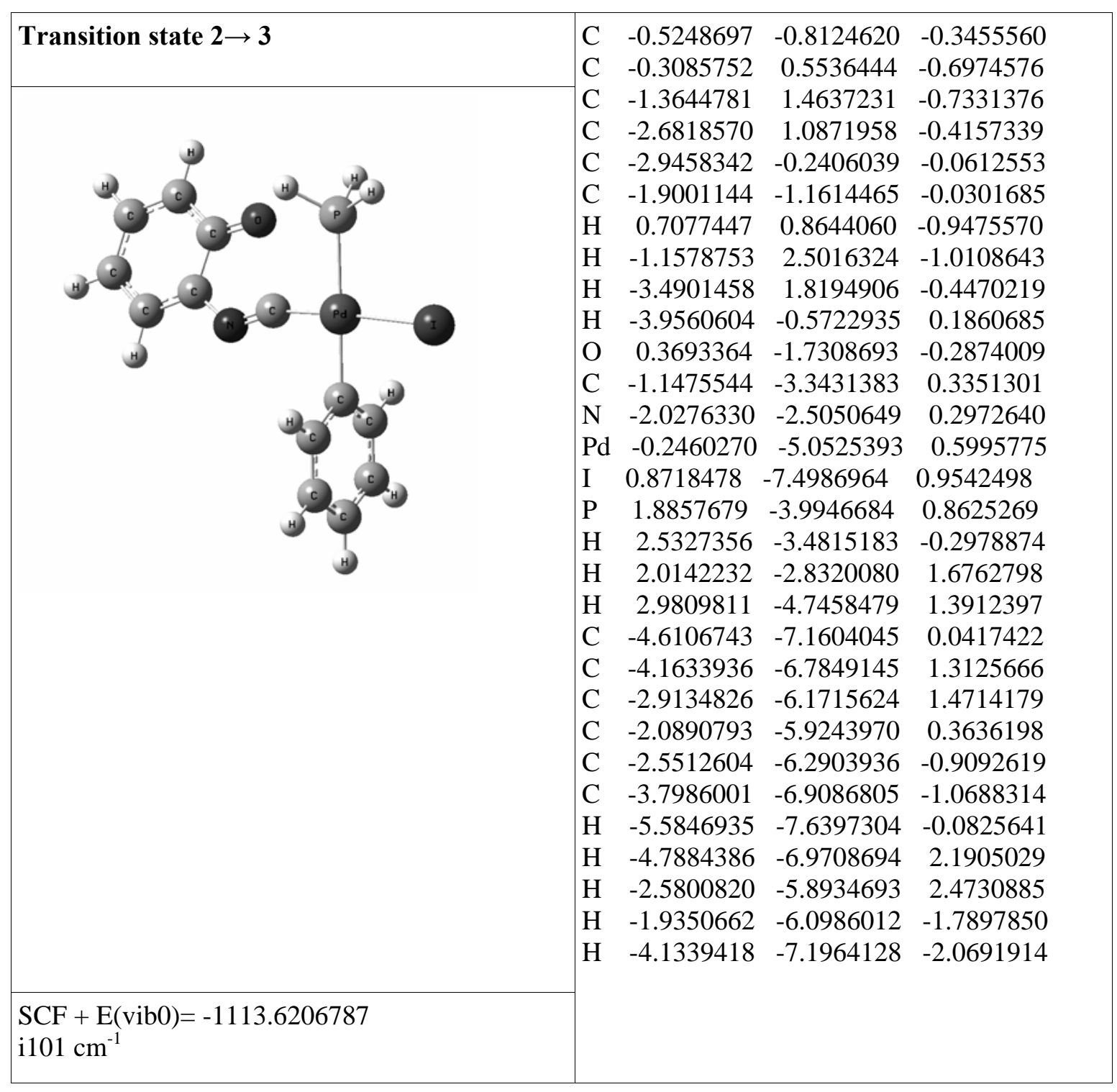

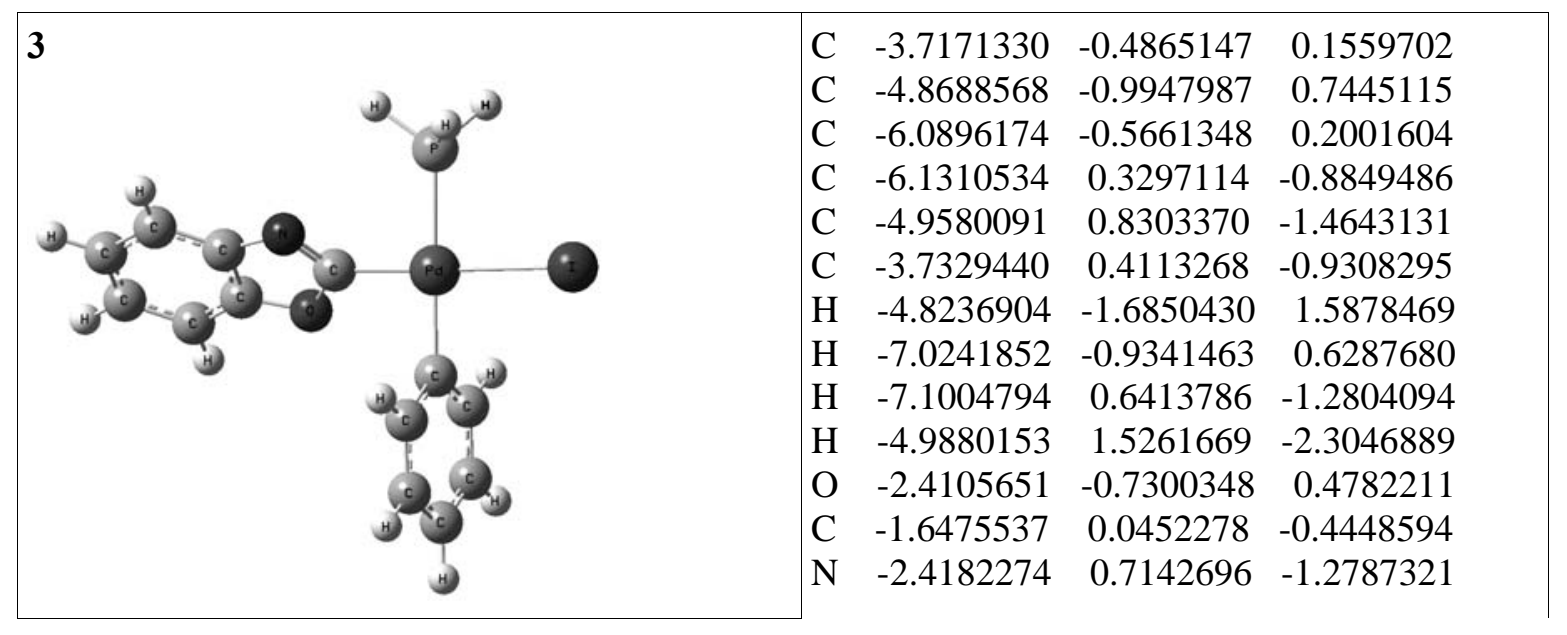




\begin{tabular}{|l|lrrr|}
\hline $\mathrm{SCF}+\mathrm{E}(\mathrm{vib})=\quad-1113.6356531$ & $\mathrm{Pd}$ & 0.3544238 & -0.0062552 & -0.4088132 \\
$\mathrm{I}$ & 3.1168992 & -0.0477573 & -0.4314467 \\
$\mathrm{P}$ & 0.4972635 & -0.0707867 & -2.7705036 \\
$\mathrm{H}$ & 1.2721691 & -1.0749227 & -3.4318969 \\
$\mathrm{H}$ & -0.6709741 & -0.1593618 & -3.5972608 \\
$\mathrm{H}$ & 1.0917696 & 1.0481203 & -3.4328675 \\
$\mathrm{C}$ & 0.2545513 & 0.3040843 & 4.4604620 \\
$\mathrm{C}$ & -0.0000526 & 1.4320104 & 3.6737737 \\
$\mathrm{C}$ & 0.0037035 & 1.3359820 & 2.2752782 \\
$\mathrm{C}$ & 0.2750377 & 0.1174099 & 1.6300628 \\
$\mathrm{C}$ & 0.5284765 & -1.0079683 & 2.4330562 \\
$\mathrm{C}$ & 0.5177780 & -0.9176596 & 3.8309518 \\
$\mathrm{H}$ & 0.2488370 & 0.3758129 & 5.5511064 \\
$\mathrm{H}$ & -0.2087868 & 2.3952172 & 4.1488600 \\
$\mathrm{H}$ & -0.2069978 & 2.2291179 & 1.6828635 \\
$\mathrm{H}$ & 0.7505344 & -1.9692946 & 1.9654729 \\
$\mathrm{H}$ & 0.7235875 & -1.8089164 & 4.4312802
\end{tabular}

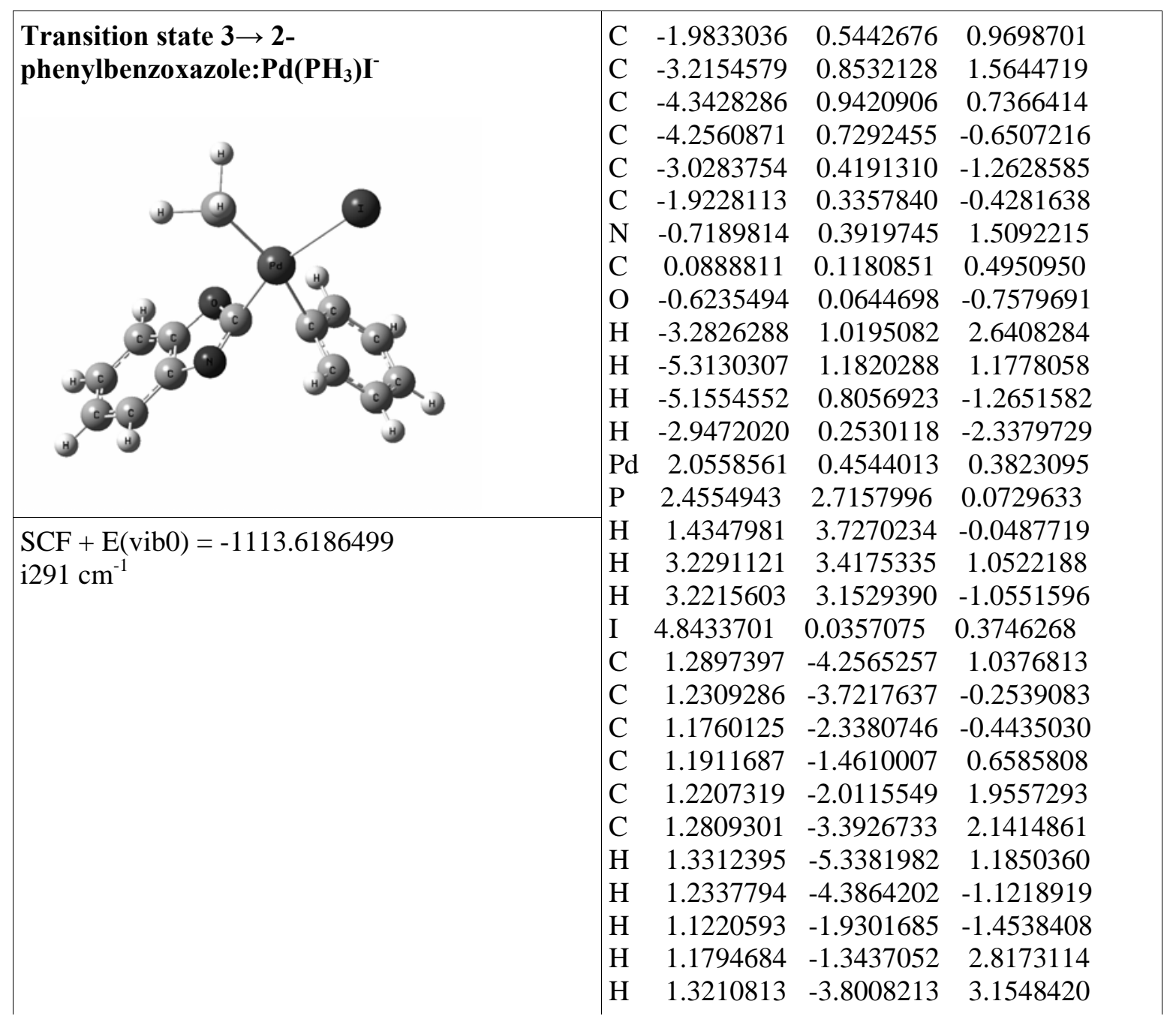




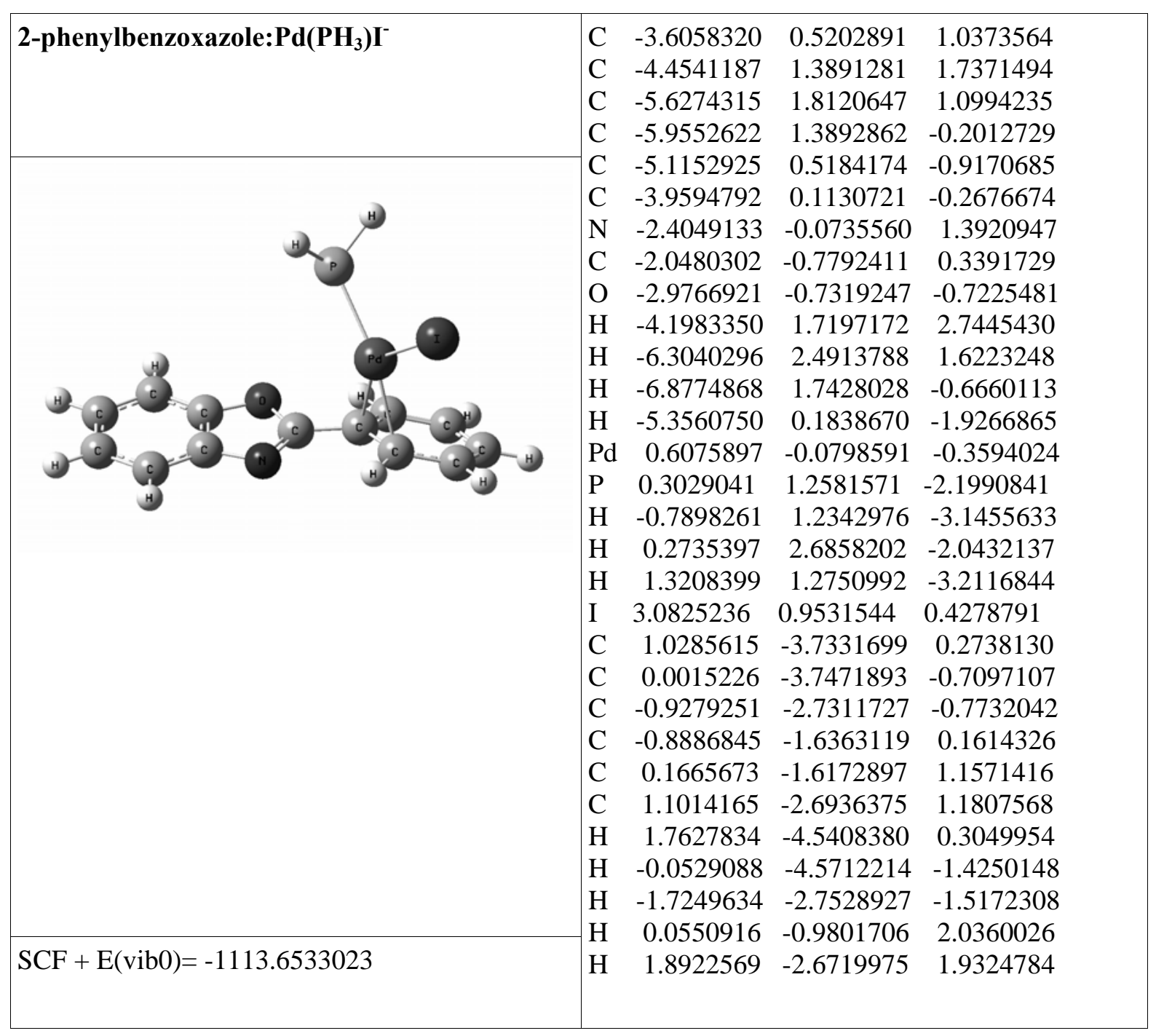

References

S1. Kunz, K. R.; Taylor, E. W.; Hutton, H. M.; Blackburn, B. J., A simple and convenient synthesis of 5-substituted benzoxazoles. Organic Preparations and Procedures International 1990, 22, (5), 613-18.

S2. Helfferich, F. G. Kinetics of Homogeneous Multistep Reactions, 2001. 